\section{Fatores associados ao nascimento pré-termo em Campina Grande, Paraíba, Brasil: um estudo caso-controle}

\author{
Factors associated with preterm birth in Campina \\ Grande, Paraíba State, Brazil: a case-control study
}

1 Departamento de Saúde,
Universidade Estadual do
Sudoeste da Bahia, Jequié,
Brasil.
2 Faculdade de Medicina,
Universidade de São Paulo,
São Paulo, Brasil.
3 Faculdade de Saúde
Pública, Universidade de São
Paulo, São Paulo, Brasill.
Instituto de Pesquisa
Professor Joaquim Amorim
Neto, Campina Grande,
Brasil.
Correspondência
P. L. Assunção
Departamento de Saúde,
Universidade Estadual do
Sudoeste da Bahia.
Rua José Moreira Sobrinho
s/n, Jequié, BA
45206-0o, Brasil.
pllisiane@grmail.com

Abstract

A case-control study (2008-2009) analyzed risk factors for preterm birth in the city of Campina Grande, Paraíba State, Brazil. A total of 341 preterm births and 424 controls were included. A multiple logistic regression model was used. Risk factors for preterm birth were: previous history of preterm birth (OR = 2.32; 95\%CI: 1.25-4.29), maternal age $(O R=2.00 ; 95 \% C I: 1.00-4.03)$, inadequate prenatal care $(O R=2.15 ; 95 \% C I$ : 1.40-3.27), inadequate maternal weight gain $(O R=2.33$; 95\%CI: 1.45-3.75), maternal physical injury (OR = 2.10; 95\%CI: 1.22-3.60), hypertension with eclampsia $(O R=17.08 ; 95 \% C I$ : 3.67-79.43) and without eclampsia $(O R=6.42$; 95\%CI: 3.50-11.76), hospitalization $(O R=5.64$; 95\%CI: 3.47-9.15), altered amniotic fluid volume $(O R=2.28 ; 95 \% C I: 1.32-3.95)$, vaginal bleeding (OR = 1.54; 95\%CI: 1.01-2.34), and multiple gestation (OR = 22.65; 95\%CI: 6.22-82.46). High and homogeneous prevalence of poverty and low maternal schooling among both cases and controls may have contributed to the fact that socioeconomic variables did not remain significantly associated with preterm birth.

Premature Birth; Risk Factors; Socioeconomic Factors
Paula Lisiane Assunção 1,2 Hillegonda Maria Dutilh Novaes 2 Gizelton Pereira Alencar 3 Adriana Suely de Oliveira Melo 4 Marcia Furquim de Almeida ${ }^{3}$

\section{Introdução}

As evidências de riscos importantes de morbimortalidade associados à prematuridade e, por conseguinte, à viabilidade perinatal tornam o nascimento pré-termo objeto destacado de pesquisa em todo o mundo 1,2,3.

Além da discussão sobre a importância do parto pré-termo como marcador para desfechos neonatais adversos, sua crescente incidência também preocupa e contribui para que seja considerado um dos principais problemas de saúde pública internacionalmente ${ }^{3}$. A incidência global de nascimento pré-termo estimada para o ano de 2005 foi de 9,6\%, e as maiores taxas foram observadas nos Estados Unidos da América (10,6\%) e em países da África (11,9\%), e as menores, em países da Europa (6,2\%). Em números absolutos, a prematuridade afeta desproporcionalmente os países em desenvolvimento, principalmente os da Ásia e África 4. No Brasil, a tendência de aumento dos pré-termos acompanha o panorama mundial. Recente revisão dos estudos nacionais de base populacional, com dados primários, mostrou aumento de $4 \%$ para $12 \%$ da prevalência, em diferentes contextos, entre 1980 e 20005.

O parto pré-termo resulta de um conjunto de fatores inter-relacionados e traz, anualmente, aos países um alto custo social e econômico, uma vez que impacta significantemente na mortalidade infantil e na qualidade de vida dos que sobrevivem com sequelas 1 . 
A compreensão do aumento sustentado do nascimento pré-termo ao lado do decréscimo da morbidade e mortalidade perinatal, em muitos países, nas últimas décadas, constitui-se em desafio atual. O conhecimento acumulado em relação aos determinantes do nascimento prétermo ainda não explica o aumento temporal nas últimas décadas, mas há consenso em relação à etiologia multifatorial e sua variabilidade em diferentes contextos, de acordo com a contribuição de diversos fatores genéticos e ambientais 6 .

O presente artigo analisou os fatores de risco para o nascimento pré-termo em partos hospitalares de mães residentes em Campina Grande, Paraíba, Brasil, contexto que representa condições socioeconômicas e de atenção à gestação e ao parto existentes em cidades de porte médio da Região Nordeste.

\section{Métodos}

Foi realizado estudo caso-controle de base populacional em que foram investigados os fatores de risco para nascimento pré-termo em nascidos vivos hospitalares de mães residentes no Município de Campina Grande. Os dados foram coletados no período de junho de 2008 a maio de 2009.

O cálculo da amostra foi realizado no OpenEpi Versão 2 (http://www.OpenEpi.com), seguindo parâmetros baseados nos resultados preliminares da pesquisa Fatores de Risco para Nascimentos Pré-Termo, desenvolvida em Londrina, Paraná, e na precisão de odds ratio (OR). O tamanho amostral definido foi de 394 recémnascidos para o grupo de casos (pré-termo, < 37 semanas de gestação) e de 394 para o grupo controle (não pré-termo, $\geq 37$ semanas de gestação). Esses números possibilitariam obter estimativas estatisticamente significantes $(\alpha=0,05$ e $1-\beta=$ 0,80 ) para OR em torno de 1,7 e para uma prevalência esperada de exposição no grupo controle de $15 \%$.

Os casos elegíveis foram todos os nascidos vivos pré-termo de partos hospitalares de mães residentes no Município de Campina Grande e ocorridos no período de referência. Os controles foram obtidos a partir de amostra dos nascidos não pré-termo estratificada por hospital e com partilha proporcional ao número de nascimento de cada unidade. A identificação e a seleção dos casos e controles elegíveis foram realizadas pela busca ativa diária nos hospitais com base na idade gestacional (IG) considerada em semanas.

A definição de casos e controles foi realizada a partir de algoritmo elaborado considerando-se a maior precisão e o uso preferencial em estudos epidemiológicos de cada medida da idade gestacional 7. A seleção final da amostra seguiu os seguintes critérios: exclusão de recém-nascidos (a) que não apresentassem IG definida em semanas, e (b) daqueles cuja classificação de prétermo e não pré-termo apresentasse divergência entre as medidas registradas; definição da IG considerando-se, por ordem de prioridade, (a) o exame de ultrassonografia realizado com menos de 20 semanas gestacionais, (b) na ausência desse registro, considerou-se a IG registrada pelo obstetra, (c) na ausência das informações anteriores, foi considerada a IG calculada a partir da data da última menstruação (DUM) referida pela mãe, e (d) havendo apenas o registro da IG feito pelo pediatra, esse era considerado.

Informações adicionais e detalhamento metodológico estão disponíveis em artigo publicado ${ }^{8}$.

A captação de casos e controles foi expandida em um pouco mais de $10 \%$ em razão da ausência de registro da IG em semanas, bem como da identificação de divergências entre as diversas fontes de registro observadas durante a coleta. Ocorreram quatro perdas entre os casos, devido à alta precoce, e nove recusas; dessas, sete eram de gestação única e duas de gemelares. Entre os controles, foram observadas 29 recusas, no entanto, essas foram substituídas mediante seleção de controle subsequente à recusa. A amostra final obtida foi de 341 casos e 424 controles.

As análises univariadas foram realizadas com 765 registros, e a regressão logística múltipla, com 641. As tabelas apresentam número variável de casos e controles em razão de alguns valores faltantes (missings), no entanto, esses não chegam a $1 \%$ em nenhuma variável.

O nascimento pré-termo foi considerado variável desfecho, e as variáveis independentes foram agrupadas em cinco blocos, conforme modelo teórico baseado em cadeia hierarquizada em três níveis - distal, intermediário e proximal 9: (a) Bloco 1 - características socioeconômicas (escolaridade, renda, ocupação, estado civil, chefe e tipo de família); (b) Bloco 2 - condições pré-concepcionais (idade, peso, altura e índice de massa corporal - IMC) e história reprodutiva (número de gestações, paridade, intervalo intergestacional); (c) Bloco 3 - características maternas e gestacionais (consumo de álcool e fumo; caminhada; planejamento e aceitação da gravidez; situações de estresse; qualidade do prénatal); (d) Bloco 4 - intercorrências maternas na gestação (hipertensão, diabetes, pré-eclampsia); (e) Bloco 5 - características fetais (tipo de parto, sexo e peso ao nascer).

Dados sobre as variáveis foram obtidos por meio de questionário aplicado às mães e de pro- 
tocolo hospitalar (prontuários obstétricos e de recém-nascidos), com instrumentos validados 10 .

O IMC foi calculado a partir do peso e altura na fase pré-concepcional e categorizado em baixo peso, peso normal, sobrepeso e obesidade, segundo os critérios da Organização Mundial da Saúde 11. O ganho ponderal gestacional foi classificado em insuficiente, adequado e excessivo, segundo as recomendações do Institute of Medicine dos Estados Unidos 12.

A assistência pré-natal foi categorizada em: pré-natal inadequado II (três ou mais variáveis com respostas negativas), pré-natal inadequado I (uma ou duas variáveis com respostas negativas), não fez pré-natal e pré-natal adequado (todas as variáveis com respostas positivas). Essas categorias foram criadas com base nos seguintes conceitos e variáveis: (1) início do pré-natal - resposta positiva: 1o, 2o ou 3o mês gestacional; (2) número de consultas de pré-natal - resposta positiva: três ou mais consultas; (3) exame de urina realizado - resposta positiva: sim; (4) exame de sangue realizado - resposta positiva: sim; (5) exame ultrassonográfico realizado - resposta positiva: sim; (6) mensuração da altura do fundo uterino realizada - resposta positiva: sim no mínimo duas vezes; (7) ausculta dos batimentos cárdio-fetais realizada - resposta positiva: sim no mínimo duas vezes; (8) verificação da pressão arterial da gestante - resposta positiva: sim no mínimo duas vezes; (9) verificação do peso da gestante - resposta positiva: sim no mínimo duas vezes; (10) IG informada à gestante em semanas - resposta positiva: sim no mínimo duas vezes; (11) orientações recebidas sobre sinais de alerta durante a gravidez - resposta positiva: sim; (12) orientações recebidas sobre como identificar o início do trabalho de parto - resposta positiva: sim.

As entrevistas com as mães foram feitas durante suas permanências no hospital; na impossibilidade, algumas foram realizadas no domicílio da mãe. A coleta de dados foi realizada por graduandos da área de saúde que cursavam, no mínimo, o segundo ano, selecionados e treinados para esse fim. Cinco por cento das entrevistas e dos formulários hospitalares foram repetidos.

A seleção final dos casos e controles e análises dos dados foram realizadas pelo pacote estatístico Stata 8.0 (Stata Corp., College Station, Estados Unidos). Foi utilizada análise de regressão logística múltipla para avaliar o ajuste das variáveis independentes e de confusão. No processo de modelagem, as variáveis de cada bloco foram incluídas segundo o menor valor de $\mathrm{p}$ (considerando-se $\mathrm{p}<0,20$ ), antes da inclusão do bloco subsequente. O método de entrada das variáveis foi o stepwise forward, no qual, em cada etapa, permaneciam, no modelo, as variáveis que apresentaram valor de $\mathrm{p} \leq 0,05$ no teste de razão de verossimilhanças. $\mathrm{O}$ efeito causal foi estudado pela análise hierarquizada, conforme anteriormente mencionado nessa seção.

O projeto de pesquisa foi aprovado pela Comissão de Ética para Análise de Projetos de Pesquisa do Hospital das Clínicas e da Faculdade de Medicina da Universidade de São Paulo/ CAPPesq (protocolo de pesquisa no. 1207/07). Foi solicitada a participação das mães na pesquisa pela assinatura de termo de consentimento livre e informado.

\section{Resultados}

O percentual de pré-termos classificados com menos de 28 semanas gestacionais foi de 9,38\%, de 28 a 31 semanas foi de $17,89 \%$, de 32 a 33 semanas foi igual a $17,3 \%$, e $55,43 \%$ foram classificados com 34 a 36 semanas gestacionais. Dentre os controles, o percentual de nascidos de 37 a 39 semanas foi de $58,02 \%$ enquanto que $5,9 \%$ foram de nascidos pós-termo (> 42 semanas).

Quanto às características socioeconômicas (Bloco 1), apresentaram valor de $\mathrm{p}<0,20$ as variáveis: escolaridade da mãe, ocupação materna, renda familiar per capita, região de moradia e migração; dessas, apenas a renda familiar per capita permaneceu significante (valor de $\mathrm{p} \leq 0,05$ ) na análise múltipla (Tabela 1). As variáveis não selecionadas para a análise de regressão múltipla foram: raça/cor materna, tipo de família, idade do responsável pelo sustento e escolaridade do responsável (dados não apresentados em tabela).

As proporções da baixa renda $\left[\chi^{2}\right.$ (1g.l.) = 1,7328; $\mathrm{p}=0,188]$, menor escolaridade [ $\chi^{2}$ (2g.l.) $=$ $3,5720 ; \mathrm{p}=0,168]$, ocupação materna [ $\chi^{2}$ (3g.l.) = $1,3651 ; \mathrm{p}=0,714]$ e região de moradia $\left[\chi^{2}\right.$ (1g.l.) $=$ $0,8885 ; \mathrm{p}=0,346]$ não se mostraram associadas ao grupo que apresentou valores faltantes.

Das variáveis pré-concepcionais (Bloco 2), idade materna, estado nutricional materno, filho anterior pré-termo, filho anterior com baixo peso ao nascer, perda de filho com menos de um ano, gravidez planejada, hipertensão arterial prévia, doença periodontal prévia e cirurgia abdominal prévia apresentaram $\mathrm{p}<0,20$ (Tabela 2). Após ajuste no bloco, permaneceram significantes idade materna e filho anterior pré-termo. Demais variáveis desse bloco que foram testadas, porém não apresentaram $\mathrm{p}<0,20$ foram: mãe nasceu pré-termo, disfunção da tireoide, asma, diabetes, número de gestações, intervalo intergestacional, cesárea anterior, tratamento para engravidar.

O maior percentual de renda per capita acima de três salários mínimos está entre as famílias 
Número e percentual de casos e controles e odds ratio (OR) bruto e ajustado, segundo variáveis selecionadas relativas às características socioeconômicas (Bloco 1) de mães residentes em Campina Grande, Paraíba, Brasil, 2008-2009.

\begin{tabular}{|c|c|c|c|c|c|c|c|c|c|c|}
\hline \multirow[t]{2}{*}{ Variáveis } & \multicolumn{2}{|c|}{ Casos } & \multicolumn{2}{|c|}{ Controles } & \multirow[t]{2}{*}{ OR bruto } & \multirow[t]{2}{*}{ IC95\% } & \multirow{2}{*}{$\begin{array}{c}\text { Valor de } \\
\qquad p^{*}\end{array}$} & \multirow{2}{*}{$\begin{array}{c}\text { OR } \\
\text { ajustado }\end{array}$} & \multirow[t]{2}{*}{ IC95\% } & \multirow[t]{2}{*}{ Valor de $p$ ** } \\
\hline & n & $\%$ & $\mathrm{n}$ & $\%$ & & & & & & \\
\hline \multicolumn{10}{|l|}{ estudo) } & 0,7402 \\
\hline $0-7$ & 134 & 39,30 & 161 & 37,97 & 0,74 & $0,47-1,13$ & & 0,79 & $0,48-2,07$ & \\
\hline $8-11$ & 145 & 42,23 & 208 & 49,06 & 0,62 & $0,40-0,93$ & & 0,96 & $0,54-1,73$ & \\
\hline 12 ou mais & 62 & 18,18 & 55 & 12,97 & 1,00 & Referência & & 1,00 & Referência & \\
\hline Ocupação materna & & & & & & & 0,1529 & & & 0,1209 \\
\hline Desempregada & 15 & 4,40 & 15 & 3,54 & 0,70 & $0,27-1,79$ & & 0,48 & $0,20-1,17$ & \\
\hline Do lar ou estudante & 218 & 63,93 & 272 & 64,15 & 0,56 & $0,30-1,04$ & & 0,71 & $0,29-1,71$ & \\
\hline Manual/semiqualificada & 81 & 23,75 & 118 & 27,83 & 0,48 & $0,24-0,93$ & & 0,57 & $0,27-1,18$ & \\
\hline Qualificada & 27 & 7,92 & 19 & 4,48 & 1,00 & Referência & & 1,00 & Referência & \\
\hline Renda familiar per capita (salários & & & & & & & 0,0258 & & & 0,092 \\
\hline \multicolumn{11}{|l|}{ mínimos) } \\
\hline 3 ou mais & 15 & 5,43 & 15 & 4,11 & 1,00 & Referência & & 1,00 & Referência & \\
\hline $1-3$ & 72 & 26,09 & 59 & 16,16 & 1,22 & $0,55-2,71$ & & 1,56 & $0,67-3,65$ & \\
\hline$<1$ & 189 & 68,48 & 291 & 79,73 & 0,65 & $0,31-1,36$ & & 0,71 & $0,30-1,68$ & \\
\hline Região de moradia & & & & & & & 0,0467 & & & 0,1107 \\
\hline Rural & 19 & 5,57 & 40 & 9,43 & 0,56 & $0,32-0,99$ & & 0,62 & $0,34-1,13$ & \\
\hline Urbana & 322 & 94,43 & 384 & 90,57 & 1,00 & Referência & & 1,00 & Referência & \\
\hline Migração & & & & & & & 0,0833 & & & 0,3271 \\
\hline Reside há menos de 2 anos & 273 & 80,06 & 317 & 74,76 & 1,35 & $0,95-1,91$ & & 1,20 & $0,33-1,13$ & \\
\hline Reside pelo menos há 2 anos & 68 & 19,94 & 107 & 25,24 & 1,00 & Referência & & 1,00 & Referência & \\
\hline
\end{tabular}

IC95\%: intervalo de 95\% de confiança.

* Teste de qui-quadrado;

** Teste de razão de verossimilhanças;

*** Teste exato de Fisher.

cujas mães têm 35 anos ou mais $(17,4 \%)$, ao passo que, entre as mães de 20-34 anos, esse percentual é de $4,2 \%$ e, entre 13 e 19 anos, de $0,76 \%$. As variáveis renda familiar per capita $\left(\chi^{2}=55,03\right.$; $\mathrm{p}<$ $0,001)$, escolaridade da mãe $\left(\chi^{2}=42,26 ; \mathrm{p}<0,001\right)$ e ocupação da mãe $\left(\chi^{2}=84,69 ; \mathrm{p}<0,001\right)$ estão associadas à idade da mãe, indicando melhores condições para aquelas acima de 35 anos. Algumas dessas variáveis apresentaram ajuste acima de $10 \%$ no bloco, no entanto, a inclusão de interações dessas três variáveis com a renda per capita não se mostrou significante [interações com escolaridade: $\chi^{2}$ (3g.l.) $=0,17 ; \mathrm{p}=0,9825$; ocupação: $\chi^{2}$ (35g.l.) = 7,49; $\mathrm{p}=0,1915$; idade: $\chi^{2}$ (3g.l.) = $2,66 ; \mathrm{p}=0,4463]$.

Dentre as variáveis de características maternas e gestacionais (Bloco 3), mostraram-se associadas ao desfecho na análise univariada: ganho ponderal materno, caminhada, dano físico materno durante a gestação, uso materno de an- tidepressivos e assistência pré-natal; dessas, foram selecionadas ganho ponderal materno, dano físico materno durante a gestação e assistência pré-natal, após o ajuste no bloco (Tabela 3). As variáveis não selecionadas foram: reside com o companheiro há, pelo menos, dois anos; tentativa de aborto; mudou de companheiro após a gestação anterior; tabagismo materno na gestação; consumo alcoólico na gestação; reações materna, paterna e familiar à gravidez (dados não apresentados em tabela).

Dos Blocos 4 e 5, selecionaram-se: sangramento vaginal, alterações do volume do líquido amniótico, hipertensão arterial na gestação, internações durante a gestação e tipo de gestação, e essas permaneceram com seus efeitos inalterados (Tabela 4). As variáveis infecção urinária na gestação e sexo do recém-nascido apresentaram p > 0,20 (dados não apresentados em tabela). No modelo final, foram incluídas e mostraram-se 
Tabela 2

Número e percentual de casos e controles e odds ratio (OR) bruto e ajustado, segundo variáveis selecionadas relativas às condições pré-concepcionais e história reprodutiva (Bloco 2) de mães residentes em Campina Grande, Paraíba, Brasil, 2008-2009.

\begin{tabular}{|c|c|c|c|c|c|c|c|c|c|c|}
\hline \multirow[t]{2}{*}{ Variáveis } & \multicolumn{2}{|c|}{ Casos } & \multicolumn{2}{|c|}{ Controles } & \multirow[t]{2}{*}{ OR bruto } & \multirow[t]{2}{*}{ IC95\% } & \multirow[t]{2}{*}{ Valor de p * } & \multirow[t]{2}{*}{ OR ajustado } & \multirow[t]{2}{*}{ IC95\% } & \multirow[t]{2}{*}{ Valor de $p$ ** } \\
\hline & $\mathbf{n}$ & $\%$ & $\mathbf{n}$ & $\%$ & & & & & & \\
\hline Idade materna (anos) & & & & & & & 0,0007 & & & 0,0023 \\
\hline 13-19 & 72 & 21,11 & 90 & 21,23 & 1,11 & $0,77-1,58$ & & 1,21 & $0,81-1,80$ & \\
\hline $20-34$ & 224 & 65,69 & 311 & 73,35 & 1,00 & Referência & & 1,00 & Referência & \\
\hline 35 ou mais & 45 & 13,20 & 23 & 5,42 & 2,71 & $1,58-4,65$ & & 2,72 & $1,52-4,87$ & \\
\hline Estado nutricional materno & & & & & & & 0,0992 & & & 0,3834 \\
\hline Baixo peso & 27 & 7,92 & 26 & 6,13 & 1,45 & $0,81-2,57$ & & 1,35 & $0,71-2,59$ & \\
\hline Adequado & 171 & 50,15 & 239 & 56,37 & 1,00 & Referência & & 1,00 & Referência & \\
\hline Sobrepeso & 55 & 16,13 & 46 & 10,85 & 1,67 & $1,07-2,59$ & & 1,34 & $0,82-2,20$ & \\
\hline Obesidade & 88 & 25,81 & 113 & 26,65 & 1,08 & $0,77-1,53$ & & 0,88 & $0,60-1,30$ & \\
\hline Filho anterior pré-termo & & & & & & & 0,0005 & & & 0,0007 \\
\hline $\operatorname{Sim}$ & 54 & 15,84 & 33 & 7,78 & 2,22 & $1,40-3,54$ & & 2,37 & $1,43-3,94$ & \\
\hline Não & 287 & 84,16 & 391 & 92,22 & 1,00 & Referência & & 1,00 & Referência & \\
\hline Filho anterior com baixo peso & & & & & & & 0,0120 & & & 0,9379 \\
\hline $\operatorname{Sim}$ & 50 & 14,66 & 36 & 8,49 & 1,79 & $1,14-2,82$ & & 1,03 & $0,50-2,13$ & \\
\hline Não & 289 & 84,75 & 387 & 91,27 & 1,00 & Referência & & 1,00 & Referência & \\
\hline $\begin{array}{l}\text { Perda de filho com menos de } \\
\text { um ano }\end{array}$ & & & & & & & 0,0768 & & & 0,2779 \\
\hline Sim & 16 & 4,69 & 10 & 2,36 & 2,03 & $0,91-4,56$ & & 1,67 & $0,65-4,25$ & \\
\hline Não & 325 & 95,31 & 414 & 97,64 & 1,00 & Referência & & 1,00 & Referência & \\
\hline Gravidez planejada & & & & & & & 0,1348 & & & 0,2845 \\
\hline Não & 216 & 63,34 & 246 & 58,02 & 1,25 & $0,93-1,67$ & & 1,20 & $0,86-1,66$ & \\
\hline $\operatorname{Sim}$ & 125 & 36,66 & 178 & 41,98 & 1,00 & Referência & & 1,00 & Referência & \\
\hline Hipertensão arterial prévia & & & & & & & 0,0680 & & & 0,1756 \\
\hline $\operatorname{Sim}$ & 30 & 8,80 & 23 & 5,42 & 1,68 & $0,95-2,95$ & & 1,54 & $0,82-2,89$ & \\
\hline Não & 311 & 91,2 & 401 & 94,58 & 1,00 & Referência & & 1,00 & Referência & \\
\hline Doença periodontal prévia & & & & & & & 0,0273 & & & 0,1495 \\
\hline $\operatorname{Sim}$ & 95 & 27,86 & 89 & 20,99 & 1,45 & $1,04-2,02$ & & 1,32 & $0,91-1,92$ & \\
\hline Não & 246 & 72,14 & 335 & 79,01 & 1,00 & Referência & & 1,00 & Referência & \\
\hline Cirurgia abdominal prévia & & & & & & & 0,0300 & & & 0,1890 \\
\hline $\operatorname{Sim}$ & 25 & 7,33 & 16 & 3,77 & 2,01 & $1,05-3,85$ & & 1,67 & $0,77-3,61$ & \\
\hline Não & 316 & 92,67 & 408 & 96,23 & 1,00 & Referência & & 1,00 & Referência & \\
\hline
\end{tabular}

IC95\%: intervalo de 95\% de confiança.

* Teste de qui-quadrado;

** Teste de razão de verossimilhanças.

associadas ao desfecho as seguintes variáveis: filho anterior pré-termo, idade da mãe, assistência pré-natal, ganho ponderal materno, dano físico materno durante a gestação, hipertensão arterial na gestação, internação durante a gestação, alteração do volume do líquido amniótico, sangramento vaginal e tipo de gestação (Tabela 5).

\section{Discussão}

Os métodos mais utilizados pelos profissionais de saúde de Campina Grande para a determinação da IG foram a DUM e a estimação clínica do obstetra. A qualidade dos registros clínicos hospitalares encontrados influiu não apenas na seleção da amostra, mas também em diversas outras medidas de variáveis de interesse, como, por exemplo, na classificação dos partos pré-termo em seus subtipos (espontâneo, com ou sem 
Número e percentual de casos e controles e razões de odds ratio (OR) bruto e ajustado, segundo variáveis selecionadas relativas às características maternas e gestacionais (Bloco 3) de mães residentes em Campina Grande, Paraíba, Brasil, 2008-2009.

\begin{tabular}{|c|c|c|c|c|c|c|c|c|c|c|}
\hline \multirow[t]{2}{*}{ Variáveis } & \multicolumn{2}{|c|}{ Casos } & \multicolumn{2}{|c|}{ Controles } & \multirow[t]{2}{*}{ OR bruto } & \multirow[t]{2}{*}{ IC95\% } & \multirow[t]{2}{*}{ Valor de $p$ * } & \multirow[t]{2}{*}{ OR ajustado } & \multirow[t]{2}{*}{ IC95\% } & \multirow[t]{2}{*}{ Valor de $p$ ** } \\
\hline & $\mathbf{n}$ & $\%$ & $\mathbf{n}$ & $\%$ & & & & & & \\
\hline Ganho ponderal materno & & & & & & & 0,0003 & & & 0,0012 \\
\hline Insuficiente & 132 & 38,71 & 107 & 25,24 & 1,70 & $1,17-2,46$ & & 1,68 & $1,13-2,60$ & \\
\hline Adequado & 97 & 28,45 & 134 & 31,60 & 1,00 & Referência & & 1,00 & Referência & \\
\hline Excessivo & 77 & 22,58 & 141 & 32,25 & 0,75 & $0,51-1,10$ & & 0,80 & $0,53-1,21$ & \\
\hline Caminhada (minutos) & & & & & & & 0,0651 & & & 0,1863 \\
\hline 30 ou mais & 48 & 14,08 & 81 & 19,10 & 0,69 & $0,46-1,02$ & & 0,73 & $0,47-1,16$ & \\
\hline Não ou menos de 30 & 293 & 85,92 & 343 & 80,90 & 1,00 & Referência & & 1,00 & Referência & \\
\hline Atividade vigorosa & & & & & & & 0,0709 & & & 0,3729 \\
\hline $\operatorname{Sim}$ & 204 & 59,82 & 226 & 53,30 & 1,30 & $0,97-1,74$ & & 1,15 & $0,83-1,60$ & \\
\hline Não & 137 & 40,18 & 198 & 46,70 & 1,00 & Referência & & 1,00 & Referência & \\
\hline Preocupações durante a gestação & & & & & & & 0,0596 & & & 0,1715 \\
\hline $\operatorname{Sim}$ & 150 & 43,99 & 158 & 37,26 & 1,32 & $0,98-1,76$ & & 1,26 & $0,91-1,75$ & \\
\hline Não & 191 & 56,01 & 266 & 62,74 & 1,00 & Referência & & 1,00 & Referência & \\
\hline $\begin{array}{l}\text { Dano físico materno durante a } \\
\text { gestação }\end{array}$ & & & & & & & 0,0001 & & & 0,0050 \\
\hline $\operatorname{Sim}$ & 66 & 19,35 & 39 & 9,20 & 2,36 & $1,54-3,64$ & & 1,94 & $1,22-3,10$ & \\
\hline Não & 275 & 80,65 & 385 & 90,8 & 1,00 & Referência & & 1,00 & Referência & \\
\hline Uso materno de antidepressivos & & & & & & & $0,0280 * \star *$ & & & 0,1026 *** \\
\hline $\operatorname{Sim}$ & 8 & 2,35 & 2 & 0,47 & 5,06 & $1,06-24,18$ & & 3,41 & $0,69-16,93$ & \\
\hline Não & 333 & 97,65 & 422 & 99,53 & 1,00 & Referência & & 1,00 & Referência & \\
\hline Assistência pré-natal & & & & & & & $<0,0001$ & & & 0,0004 \\
\hline Inadequada I & 91 & 26,69 & 188 & 44,34 & 1,00 & Referência & & 1,00 & Referência & \\
\hline Inadequada II & 235 & 68,91 & 233 & 54,95 & 2,08 & $1,52-2,85$ & & 1,81 & $1,30-2,51$ & \\
\hline Não fez & 15 & 4,40 & 3 & 0,71 & 10,32 & $2,78-38,25$ & & $\#$ & $\#$ & \\
\hline
\end{tabular}

IC95\%: intervalo de 95\% de confiança.

* Teste de qui-quadrado;

** Teste de razão de verossimilhanças;

$\star \star \star$ Teste exato de Fisher;

\# Categoria com poucos registros incluída na categoria inadequado II.

rompimento prematuro de membranas, e indicado pelo médico), classificação essa considerada como importante para a compreensão dos fatores de risco para nascimentos pré-termo 1,2,13.

A distribuição da IG, entre os casos, mostrou um maior número de pré-termos tardios e, entre os controles, um maior número de nascidos com 39 semanas, o que vai ao encontro dos achados mais recentes sobre o tema. Considerações adicionais sobre essa distribuição foram apresentadas em artigo anterior 8 .

No que se refere aos fatores de risco, buscouse investigar associações plausíveis e significantes na cadeia multifatorial, e foram abordadas variáveis relacionadas aos aspectos biológicos, socioeconômicos e psicossociais, segundo uma proposta de análise hierarquizada. Entre as variáveis utilizadas para mensuração das condições socioeconômicas, algumas estão sempre presentes nos estudos, tais como renda, escolaridade materna e ocupação. Atualmente, tem sido dada ênfase à inclusão, na análise, também de fatores denominados psicossociais, que buscam representar aspectos outros presentes na construção das condições de vida e que contribuem para a complexidade e variabilidade da dimensão social.

Neste estudo, os resultados encontrados na análise das variáveis socioeconômicas exigem análise cuidadosa. A baixa renda familiar per capita não se mostrou associada aos nascimentos pré-termo, possivelmente devido às caracterís- 
Número e percentual de casos e controles e razões de odds ratio (OR) bruto e ajustado, segundo variáveis selecionadas relativas às intercorrências maternas na gestação (bloco 4) e às características fetais (bloco 5) de mães residentes em Campina Grande, Paraíba, Brasil, $2008-2009$.

\begin{tabular}{|c|c|c|c|c|c|c|c|c|c|c|}
\hline \multirow[t]{2}{*}{ Variáveis } & \multicolumn{2}{|c|}{ Casos } & \multicolumn{2}{|c|}{ Controles } & \multirow[t]{2}{*}{ OR bruto } & \multirow[t]{2}{*}{ IC95\% } & \multirow[t]{2}{*}{ Valor de $p$ * } & \multirow[t]{2}{*}{ OR ajustado } & \multirow[t]{2}{*}{ IC95\% } & \multirow[t]{2}{*}{ Valor de $p$ ** } \\
\hline & $\mathbf{n}$ & $\%$ & $\mathbf{n}$ & $\%$ & & & & & & \\
\hline \multicolumn{11}{|l|}{ Bloco 4} \\
\hline Hipertensão arterial na & & & & & & & $<0,0001$ & & & $<0,0001$ \\
\hline \multicolumn{11}{|l|}{ gestação } \\
\hline Sim, com eclampsia & 14 & 4,91 & 2 & 0,55 & 11,28 & $2,48-51,26$ & & 11,74 & $2,58-53,45$ & \\
\hline Sim, sem eclampsia & 57 & 20,00 & 19 & 5,19 & 4,83 & $2,75-8,49$ & & 5,03 & $2,85-8,86$ & \\
\hline Não & 214 & 75,09 & 345 & 94,26 & 1,00 & Referência & & 1,00 & Referência & \\
\hline Internação durante a gestação & & & & & & & $<0,0001$ & & & $<0,0001$ \\
\hline $\operatorname{Sim}$ & 126 & 36,95 & 36 & 8,49 & 6,31 & $4,09-9,74$ & & 6,96 & $4,31-11,23$ & \\
\hline Não & 215 & 63,05 & 388 & 91,51 & 1,00 & Referência & & 1,00 & Referência & \\
\hline Alteração do volume do & & & & & & & $<0,0001$ & & & $<0,0001$ \\
\hline \multicolumn{11}{|l|}{ líquido amniótico } \\
\hline Sim & 79 & 23,17 & 38 & 8,96 & 3,23 & $2,10-4,96$ & & 3,86 & $2,38-6,24$ & \\
\hline Não & 247 & 72,43 & 384 & 90,57 & 1,00 & Referência & & 1,00 & Referência & \\
\hline Sangramento vaginal & & & & & & & $<0,0001$ & & & 0,0001 \\
\hline $\operatorname{Sim}$ & 128 & 37,54 & 87 & 20,52 & 2,32 & $1,67-3,23$ & & 2,03 & $1,41-2,91$ & \\
\hline Não & 213 & 62,46 & 337 & 79,48 & 1,00 & Referência & & 1,00 & Referência & \\
\hline \multicolumn{11}{|l|}{ Bloco 5} \\
\hline Tipo de gestação & & & & & & & $<0,0001$ & & & $<0,0001$ \\
\hline Múltipla & 41 & 12,02 & 7 & 1,65 & 8,14 & $3,53-18,76$ & & 15,26 & $4,46-52,23$ & \\
\hline Única & 300 & 87,98 & 417 & 98,35 & 1,00 & Referência & & 1,00 & Referência & \\
\hline
\end{tabular}

IC95\%: intervalo de 95\% de confiança.

* Teste de qui-quadrado;

** Teste de razão de verossimilhanças.

ticas da população de estudo, em que há elevada proporção de baixa renda (mais de $70 \%$ ) tanto para os casos como para os controles. Resultados distintos foram observados em estudos caso-controle sobre nascimentos pré-termo realizados em Londrina e no Rio de Janeiro 14, onde se verificou existir diferenciais da proporção de nascimentos de famílias de baixa renda entre casos e controles. Essa condição pode ter contribuído para essa variável não apresentar poder de discriminação entre casos e controles. Motivo semelhante pode ser atribuído ao comportamento da variável escolaridade materna, que se diferencia do que foi encontrado em Pelotas 15 . Alguns estudos têm mostrado que a qualidade de moradia precária 14 ou em núcleos de favela 16 estava associada aos nascimentos pré-termo. Porém, neste estudo, o local de moradia não foi testado em razão da qualidade dos dados, pois pouquíssimas mães responderam "favela".

Os resultados para as variáveis socioeconômicas em estudos realizados na América do
Norte e na Europa mostraram, em sua maioria, maior risco para baixa condição socioeconômica, principalmente medida pela renda ou escolaridade 1,17,18. Por outro lado, em estudo realizado em Santiago, no Chile (América Latina), país com perfil socioeconômico mais próximo ao do Brasil, as magnitudes desses efeitos foram mais modestas. Uma das potenciais explicações atribuídas pelos autores foi a distribuição igualitária do cuidado pré-natal que pode ter reduzido a importância dos efeitos das desigualdades sociais 19 . Entretanto, a mensuração da eficácia do cuidado pré-natal na prevenção do parto pré-termo, bem como a compreensão do seu efeito mediador 20 , tem se mostrado complexa.

$\mathrm{O}$ efeito do contexto social sobre o nascimento pré-termo ocorre de forma complexa, acumulativa, articulada e interativa e resulta da articulação entre múltiplas características individuais e contextuais, construindo condições de vida diferenciadas nos indivíduos para contextos gerais semelhantes 18 . A condição de vida 
Modelo final da regressão logística múltipla, odds ratio (OR) ajustados, com intervalo de $95 \%$ de confiança (IC95\%), e valores de p. Campina Grande, Paraíba, Brasil, 2008-2009.

\begin{tabular}{|c|c|c|c|}
\hline Variáveis & OR ajustado & IC95\% & Valor de $\mathrm{p}$ * \\
\hline \multicolumn{4}{|l|}{ Bloco 2} \\
\hline Filho anterior pré-termo & & & $<0,001$ \\
\hline $\operatorname{Sim}$ & 2,19 & $1,19-4,03$ & \\
\hline Não & 1,00 & Referência & \\
\hline Idade da mãe (anos) & & & 0,05 \\
\hline $20-34$ & 1,00 & Referência & \\
\hline $13-19$ & 1,28 & $0,80-2,04$ & \\
\hline 35 ou mais & 2,00 & $1,00-4,03$ & \\
\hline \multicolumn{4}{|l|}{ Bloco 3} \\
\hline Assistência pré-natal & & & $<0,0001$ \\
\hline Inadequada I & 1,00 & Referência & \\
\hline Inadequada II & 2,06 & $1,35-3,12$ & \\
\hline Ganho ponderal materno & & & $<0,001$ \\
\hline Insuficiente & 2,32 & $1,44-3,72$ & \\
\hline Adequado & 1,00 & Referência & \\
\hline Excessivo & 0,64 & $0,38-1,07$ & \\
\hline Ignorado & 0,71 & $0,32-1,58$ & \\
\hline Dano físico materno durante a gestação & & & $<0,001$ \\
\hline $\operatorname{Sim}$ & 2,00 & $1,17-3,42$ & \\
\hline Não & 1,00 & Referência & \\
\hline \multicolumn{4}{|l|}{ Bloco 4} \\
\hline Hipertensão arterial na gestação & & & $<0,001$ \\
\hline Sim, com eclampsia & 12,12 & $2,41-61,05$ & \\
\hline Sim, sem eclampsia & 4,41 & $2,40-8,63$ & \\
\hline Não & 1,00 & Referência & \\
\hline Internação durante a gestação & & & $<0,001$ \\
\hline Sim & 4,41 & $2,65-7,31$ & \\
\hline Não & 1,00 & Referência & \\
\hline Alteração do volume do líquido amniótico & & & 0,004 \\
\hline $\operatorname{Sim}$ & 2,26 & $1,29-3,96$ & \\
\hline Não & 1,00 & Referência & \\
\hline Sangramento & & & 0,05 \\
\hline Sim & 1,54 & $1,00-2,37$ & \\
\hline Não & 1,00 & Referência & \\
\hline \multicolumn{4}{|l|}{ Bloco 5} \\
\hline Tipo de gestação & & & $<0,001$ \\
\hline Múltipla & 25,40 & $7,07-91,34$ & \\
\hline Única & 1,00 & Referência & \\
\hline
\end{tabular}

* Teste de razão de verossimilhanças.

material materna e familiar parece interferir na saúde perinatal de forma mais ou menos imediata, mediada principalmente por estresse psicossocial, diferenças no acesso e uso de cuidado pré-natal, ausência de suporte social e adoção de comportamentos não saudáveis 1 . É necessário considerar o nascimento pré-termo, portanto, como um problema de saúde ocorrendo em contextos sociais complexos, em que se sobrepõem dimensões sociais, psicossociais e biomédicas, com articulações difíceis de serem mensuradas de forma completa e abrangente. 
No que diz respeito às características da mãe, idade de 35 anos ou mais se constituiu em risco. No entanto, no processo de modelagem, seu efeito foi reduzido pela introdução das variáveis hipertensão na gestação e tipo de gestação. A associação com idade menor do que 20 anos não foi significante.

Achados de risco para os extremos da idade materna vêm sendo referidos na literatura científica 1,13,21. A comparabilidade, entretanto, fica dificultada pela utilização de faixas etárias diferentes nas análises. Dentre os estudos brasileiros, observou-se, na coorte de nascimento de 1982 de Pelotas, risco tanto para mães jovens como para aquelas com mais de 30 anos 22. Na coorte de 2004, a categoria 35 anos ou mais foi utilizada como referência ${ }^{15}$. No estudo de São Luís, Maranhão, a idade abaixo de 18 anos, comparada com 20-24 anos, mostrou risco significante, porém, não foi observada significância para a categoria acima de 24 anos 23 . No caso-controle de Londrina, foram empregados os mesmos pontos de corte utilizados aqui, no entanto, a idade não se mostrou associada 10 .

Ser mãe jovem mostra-se associado ao aumento das taxas de parto pré-termo espontâneo, ao passo que ser mãe com mais idade mostra-se associado ao aumento das taxas de pré-termos nascidos de partos induzidos, com indicação resultante da presença de complicações gestacionais 1,3,13; neste estudo, a presença dessa indicação foi apontada pelas variáveis hipertensão e gestação múltipla.

A idade avançada compõe o quadro explicativo para o aumento das taxas de prematuridade nas sociedades desenvolvidas, como resultante das modificações observadas na vida moderna em que a mulher vem cada vez mais conquistando o mercado de trabalho e investindo na sua carreira e postergando a maternidade. Essas mulheres são também primíparas e com mais de 12 anos de escolaridade 24. Esse ainda não é o perfil epidemiológico dominante entre as gestantes de Campina Grande, todavia, parece estar se iniciando uma mudança nessa direção.

De forma semelhante à idade avançada, o aumento do uso de tecnologias para reprodução assistida também tem sido referido como explicação para o aumento temporal da prematuridade 1 . A presença de tratamento para engravidar foi testada neste trabalho, porém não foi observada sua associação com o desfecho na análise univariada (dado não apresentado em tabela), e poucas mães referiram a sua realização. Em Londrina, as mães que se submeteram a tratamentos para engravidar, mais numerosas, apresentaram uma chance para nascimento pré-termo de cerca de oito vezes maior do que aquelas que não se submeteram a esse tipo de tratamento 16 .

Para as mães não primíparas, ter filho prétermo anterior à gestação atual representou risco. Esse efeito é consistente com os observados no Sul do país 15,16. O parto anterior pré-termo tem sido utilizado como preditor clínico para risco de recorrência de parto prematuro, tanto espontâneo como indicado. O risco aumenta diretamente com o número de pré-termos prévios e é muito mais alto para recorrências após parto prematuro indicado 1,25. No estudo de Londrina, a análise dos fatores de risco não foi realizada por subtipo de pré-termo, entretanto, foram observados $41 \%$ de partos prematuros indicados entre os casos 16. Esses resultados apontam para a importância da compreensão da participação das condutas clínicas obstétricas na ocorrência de nascimentos pré-termo.

Em relação às características gestacionais, o ganho ponderal insuficiente mostrou risco para prematuridade. Estudos mostram que tanto o baixo peso pré-gestacional como o ganho ponderal insuficiente estão associados para ambos os subtipos de partos pré-termo, por sua vez, o primeiro é forte fator de risco para o segundo 13,26. Estudo realizado em Taubaté (São Paulo) verificou associação significante com ganho inferior a $13 \mathrm{~kg} 27$, e, no estudo de Londrina, observou-se risco para mães com baixo IMC pré-gestacional 16 . $\mathrm{O}$ baixo peso materno ainda é uma realidade em Campina Grande, apesar de estar em curso uma transição nutricional. Resultados de uma coorte de gestantes mostraram que coexistem o baixo peso (23\%) e o sobrepeso/obesidade (28\%) prégestacionais, bem como o ganho ponderal insuficiente $(22,6 \%)$ e excessivo $(44,2 \%)$ observados no terceiro trimestre gestacional 28 . Nessa coorte, foi observado também maior percentual de baixo peso e de peso insuficiente ao nascer 29.

Maior risco de prematuridade foi observado entre as mães que não receberam cuidado prénatal ou receberam de forma inadequada. Esse resultado foi semelhante ao observado em outros estudos brasileiros 15,16,19 e de outros países 2,13, em diferentes contextos sociais, apesar das realidades socioeconômicas diversas e da utilização de medidas diferentes. Chamam atenção o alto percentual de ausência de pré-natal (4,4\% entre os casos) e a ausência de cuidados adequados, segundo definição adotada em que foram considerados, além do início e números de consultas, a realização de procedimentos básicos. Utilizando os mesmos critérios, o estudo caso-controle de Londrina mostrou resultados muito melhores: em $2,1 \%$ dos casos, não foi realizado pré-natal, e, em $63,1 \%$, o cuidado foi adequado 16 . 
Ainda em relação ao cuidado pré-natal prestado no município, estudo mostrou que, no período de maio a junho de 2009 , dentre os $78,6 \%$ dos partos ocorridos $(\mathrm{N}=416)$, em $88 \%$, o preenchimento da curva ganho de peso/idade gestacional do cartão de acompanhamento do pré-natal estava ausente. Das parturientes que portavam seus cartões de pré-natal, $82 \%$ tiveram assistência ao parto pelo SUS, e 18\% tiveram assistência particular/convênio ou mista (SUS com adicional particular). Para essas últimas, o risco para a ausência da curva de ganho de peso no cartão foi bastante alto $(\mathrm{OR}=10,09$; IC95\%: 1,32-77,02) 30 .

O dano físico sofrido durante a gestação se mostrou significante após ajuste no bloco e ao final da modelagem. A associação entre violência física e a prematuridade é também observada em outros contextos 14. Em Londrina, a variável dano físico não foi testada, e o risco associado com situações estressantes foi captado pela variável preocupações durante a gestação 16 . No presente estudo, a associação com essa variável não se mostrou significante na análise univariada. Uma explicação possível para essa diferença pode estar nas mesmas considerações discutidas para a variável renda. Ou seja, semelhante à distribuição da renda, a presença de preocupações durante a gestação foi alta e próxima entre casos $(43,99 \%)$ e controles $(37,26 \%)$. As situações de estresse determinadas por problemas financeiros, de saúde, de trabalho ou de relacionamentos familiares, por exemplo, parecem refletir conflitos relacionados à situação socioeconômica homogeneamente desfavorável, característica do contexto estudado.

O mesmo comportamento observado nas variáveis renda e escolaridade foi observado na variável mãe reside com companheiro. O estudo de Londrina observou risco para mães que residem com seus companheiros há menos de dois anos 16, porém, em Campina Grande, essa variável não mostrou associação na análise univariada (dado não apresentado em tabela). As distribuições dessa categoria entre casos e controles foram similares, $34 \%$ e $36 \%$ respectivamente. Como parte da dimensão psicossocial, aspectos relacionados ao suporte social e emocional vêm sendo investigados nos estudos de desfechos neonatais adversos 1 .

Diferentemente do observado nos resultados para os fatores socioeconômicos e psicossociais, os resultados dos estudos das associações dos fatores obstétricos com o nascimento pré-termo são semelhantes, na maioria dos estudos 1, principalmente quanto mais estabelecidas as relações causais das variáveis com o desfecho, na perspectiva clínica e patogênica.
Vários problemas de saúde durante a gestação e parto são relacionados consistentemente a partos pré-termo espontâneo e indicado, como trabalho de parto idiopático, gestação múltipla e hipertensão na gestação 5,31.

Neste trabalho, mostraram-se significantes hipertensão arterial com ou sem eclampsia, internação hospitalar, alteração do líquido amniótico, sangramento vaginal e gestação múltipla (aqui considerada como característica fetal). Associações semelhantes foram observadas em estudos desenvolvidos no Brasil 15,16. Sangramento vaginal e alteração do líquido amniótico apresentaram magnitudes de cerca de menos da metade, e a hipertensão arterial na gestação apresentou magnitude maior, principalmente hipertensão arterial sem eclampsia. Quanto às internações hospitalares, os achados sugerem que, na falta de acompanhamento adequado das gestações de risco, as intercorrências são assistidas como urgências ou emergências em serviços terciários, levando a maior número de internações.

As taxas de descolamento prematuro de placenta são referidas como tendo aumentado nos últimos anos em países desenvolvidos e se apresentam pela presença de sangramento. Os fatores causais são vários e incluem-se a hipertensão gestacional, a gestação múltipla, o oligo-hidrâmnio e a ruptura prematura das membranas ovulares ${ }^{32}$. Estudo retrospectivo realizado em São Paulo comparou os casos ocorridos de descolamento prematuro de placenta nos períodos de 1994-1997 e de 2001-2005 e mostrou maior proporção de descolamento prematuro de placenta entre as mulheres que não realizaram pré-natal no período de 1994-1997 e entre as que apresentaram maior número de intercorrências clínicas ou obstétricas no período de 2001-2005 33 . Ressalta-se, no entanto, que o estudo foi desenvolvido em um hospital de referência para gestação de alto risco e pode ter ocorrido maior representação desse tipo de gestação.

Os dados hospitalares relacionados às intercorrências gestacionais não puderam ser utilizados em decorrência do alto percentual de não preenchimento dos registros. Dois hospitais privados, em particular, apresentaram condições muito graves, não havendo nem mesmo registro da duração da gestação em semanas. Sendo assim, para as variáveis alteração do líquido amniótico e internações durante a gestação, informadas pela mãe, pode ter ocorrido algum impacto nas medidas de efeito encontradas, dado que as informações maternas sobre complicações gestacionais podem ser menos precisas 34 . A qualidade da informação pode ser afetada pela falta de compreensão, por parte da gestante, dos esclarecimentos prestados sobre suas condições de 
saúde durante o pré-natal ou pela ausência de esclarecimentos.

No presente estudo, foram fatores de risco: idade materna de 35 ou mais, pré-termo prévio, pré-natal inadequado, ganho ponderal materno insuficiente, dano físico materno, hipertensão arterial com eclampsia e sem eclampsia, internação, alteração do volume amniótico, sangramento vaginal e gestação múltipla.

A prematuridade difere entre as populações e isso se dá em função da complexidade e da diversidade dos contextos sociais. Em Campina Grande, a pobreza e suas repercussões sobre renda, escolaridade e qualidade da atenção ainda desempenham papel importante no nascimento pré-termo, observando-se, no entanto, o início de uma transição demográfica e social entre as gestantes.

A elevada prevalência da pobreza e baixa escolaridade, retratando condições socioeconômicas homogeneamente desfavoráveis tanto nos casos como nos controles, maior que em estudos realizados na Região Sudeste, pode ter levado a menor discriminação entre os eventos estudados e contribuído para o resultado observado. Estudos adicionais, complementares, são necessários para o aprofundamento do conhecimento sobre a complexidade das cadeias causais no parto prétermo em diferentes contextos e a diferenciação pelos subtipos, espontâneo e indicado.

\section{Resumo}

Estudo caso-controle (2008-2009) de base populacional que analisou fatores de risco para nascimento prétermo em Campina Grande, Paraíba, Brasil. Foram incluídos 341 nascimentos pré-termo e 424 controles. Utilizou-se regressão logística múltipla. Foram fatores de risco: idade materna de 35 ou mais $(O R=2,00$; IC95\%: 1,00-4,03), pré-termo prévio $(O R=2,32$; IC95\%: 1,25-4,29), pré-natal inadequado $(O R=2,15$; IC95\%: $1,40-3,27)$, ganho ponderal materno insuficiente $(O R=2,33$; IC95\%: 1,45-3,75), dano físico materno $(O R=2,10 ;$ IC95\%: 1,22-3,60), hipertensão arterial com eclampsia $(O R=17,08$; IC95\%: 3,67-79,43) e sem eclampsia $(O R=6,42$; IC95\%: 3,50-11,76), internação $(O R=5,64 ;$ IC95\%: 3,47-9,15), alteração do volume amniótico (OR = 2,28; IC95\%: 1,32-3,95); sangramento vaginal (OR = 1,54; IC95\%: 1,01-2,34) e gestação múltipla $(O R=22,65$; IC95\%: 6,22-82,46). Elevada e homogênea prevalência de pobreza e baixa escolaridade pode ter contribuído para que as variáveis socioeconômicas não permanecessem com associação significativa para o nascimento pré-termo.

Nascimento Prematuro; Fatores de Risco; Fatores Socioeconômicos

\section{Colaboradores}

P. L. Assunção, H. M. D. Novaes e M. F. Almeida contribuíram na concepção do projeto, interpretação dos dados, redação do artigo e revisão crítica relevante do conteúdo intelectual e aprovação final da versão a ser publicada. P. L. Assunção realizou a análise dos dados. G. P. Alencar colaborou na análise e interpretação dos dados, revisão crítica e aprovação final da versão a ser publicada. A. S. O. Melo contribuiu com a revisão crítica e aprovação final da versão a ser publicada. 


\section{Referências}

1. Committee on Understanding Premature Birth and Assuring Healthy Outcomes Board on Health Siences Policy, Institute of Medicine. Preterm birth: causes, consequences, and prevention. Washington DC: National Academies Press; 2007.

2. Goldenberg RL, Culhane JF, Iams JD, Romero R. Epidemiology and causes of preterm birth. Lancet 2008; 371:75-84.

3. Lawn JE, Gravett MG, Nunes TM, Rubens CE, Stanton C; GAPPS Review Group. Global report on preterm birth and stillbirth ( 1 of 7): definitions, description of the burden and opportunities to improve data. BMC Pregnancy Childbirth 2010; 10 Suppl 1:S1.

4. Beck S, Wojdyla D, Say L, Betran AP, Merialdi M, Requejo JH, et al. The worldwide incidence of preterm birth: a systematic review of maternal mortality and morbidity. Bull World Health Organ 2010; 88:31-8.

5. Silveira MF, Santos A, Barros AJD, Matijasevich A, Barros FC, Victora CG. Aumento da prematuridade no Brasil: revisão de estudos de base populacional. Rev Saúde Pública 2008; 42:957-64.

6. Gravett MG, Rubens CE, Nunes TM; GAPPS Review Group. Global report on preterm birth and stillbirth ( 2 of 7): discovery science. BMC Preganancy Childbirth 2010; 10 Suppl 1:S2.

7. Ananth CV. Menstrual versus clinical estimate of gestational age dating in the United States: temporal trends and variability in indices of perinatal outcomes. Paediatr Perinatal Epidemiol 2007; 21 Suppl 2:22-30.

8. Assunção PL, Novaes HMD, Alencar GP, Melo ASO, Almeida MF. Desafios na definição da idade gestacional em estudos populacionais sobre parto prétermo: o caso de um estudo em Campina Grande (PB). Rev Bras Epidemiol 2011; 14:455-66.

9. Victora CG, Huttly SR, Fuchs SC, Olinto MTA. The role of conceptual frameworks in epidemiological analysis: a hierarchical approach. Int J Epidemiol 1997; 26:224-7.

10. Silva AMR. Fatores de risco para nascimentos prétermo no Município de Londrina - Paraná. São Paulo, 2008 [Tese de Doutorado]. São Paulo: Faculdade de Saúde Pública, Universidade de São Paulo; 2008.

11. World Health Organization. Obesity - preventing and managing the global epidemic. WHO Consultation on Obesity. Geneva: World Health Organization; 1998.

12. Subcommittee on Nutritional Status and Weight Gain During Pregnancy, Institute of Medicine. Nutrition during pregnancy: part I: weight gain, part II: nutrient supplements. Washington DC: National Academy Press; 1990.

13. Ip M, Peyman E, Lohsoonthorn V, Williams MA. A case-control study of preterm delivery risk factors according to clinical subtypes and severity. J Obstet Gynaecol Res 2010; 36:34-44.
14. Vettore MV, Gama SG, Lamarca GA, Schilithz AO, Leal MC. Housing conditions as a social determinant of low birthweight and preterm low birthweight. Rev Saúde Pública 2010; 44:1021-31.

15. Silveira MF, Victora CG, Barros AJD, Santos IS, Matijasevich A, Barros FC. Determinants of preterm birth: Pelotas, Rio Grande do Sul State, Brazil, 2004 birth cohort. Cad Saúde Pública 2010; 26 : 185-94.

16. Silva AMR, Almeida MF, Matsuo T, Soares DA. Fatores de risco para nascimentos pré-termo em Londrina, Paraná, Brasil. Cad. Saúde Pública 2009; 5:2125-38.

17. Morgen CS, Bjørk C, Andersen PK, Mortensen1LH, Andersen AN. Socioeconomic position and the risk of preterm birth - a study within the Danish National Birth Cohort. Int J Epidemiol 2008; 37:1109-20.

18. Reagan PB, Salsberry PJ. Race and ethnic differences in determinants of preterm birth in the USA: broadening the social context. Soc Sci Med 2005; 60:2217-28.

19. Kaufman JS, Alonso FT, Pino P. Multi-level modeling of social factors and preterm delivery in Santiago de Chile. BMC Pregnancy Childbirth 2008; 8:46.

20. Barros FC, Bhutta ZA, Batra M, Hansen TN, Victora CG, Rubens CE, et al. Global report on preterm birth and stillbirth (3 of 7): evidence for effectiveness of interventions. BMC Pregnancy Childbirth 2010; 10 Suppl 1:S3

21. Jolly MC, Sebire N, Harris J, Robinson S, Regan L. Obstetric risks of pregnancy in women less than 18 years old. Obstet Gynecol 2000; 96:962-6.

22. Barros FC, Huttly SR, Victora CG, Kirkwood BR, Vaughan JP. Comparison of the causes and consequences of prematurity and intrauterine growth retardation: a longitudinal study in southern Brazil. Pediatrics 1992; 902(2 Pt 1):238-44.

23. Aragão VMF, Silva AAM, Aragão LF, Barbieri MA, Coimbra LC, Ribeiro VS. Risk factors for preterm births in São Luís, Maranhão, Brazil. Cad Saúde Pública 2004; 20:57-63.

24. Branum AM, Schoendorf KC. The influence of maternal age on very preterm birth of twins: differential effects by parity. Paediatr Perinat Epidemiol 2005; 19:399-404.

25. Ananth CV, Getahun D, Peltier MR, Salihu HM, Vintzileos AA. Recurrence of spontaneous versus medically indicated preterm birth. Am J Obstet Gynecol 2006; 195:643-50.

26. Wise LA, Palmer JR, Heffner LJ, Rosenberg L. Prepregnancy body size, gestational weight gain, and risk of preterm birth in African-American women. Epidemiology 2010; 21:243-52.

27. Nascimento LFC. Epidemiology of preterm deliveries in Southeast Brazil: a hospitalbased study. Rev Bras Saúde Matern Infant 2001; 1:263-8. 
28. Assunção PL, Melo ASOM, Gondim SSR, Benício MHD, Amorim MMR, Cardoso MAA. Ganho ponderal e desfechos gestacionais em mulheres atendidas pelo Programa de Saúde da Família em Campina Grande, PB (Brasil). Rev Bras Epidemiol 2007; 10:352-60.

29. Melo ASO, Assunção PL, Gondim SSR, Carvalho DF, Amorim MMR, Benício MHD, et al. Estado nutricional materno, ganho de peso gestacional e peso ao nascer. Rev Bras Epidemiol 2007; 10:249-57.

30. Araújo-Silva ALM, Porto RF, Ferraz RP, Costa KNF, Vânia ML, Almeida CDA, et al. Preenchimento da curva de ganho de peso/idade gestacional no cartão da gestante em Campina Grande/PB [CDROM]. In: Anais do IX Congresso Brasileiro de Saúde Coletiva. Recife: ABRASCO; 2009.

31. Slattery MM, Geary M, Morrison JJ. Obstetric antecedents for preterm delivery. J Perinat Med 2008; 36:306-9.
32. Ananth CV, Oyelese Y, Srinivas N, Yeo L, Vintzileos AM. Preterm premature rupture of membranes, intrauterine infection, and oligohydramnios: risk factors for placental abruption. Obstet Gynecol 2004; 104:71-7.

33. Nomura RMY, Cabar FR, Machado TRS, Martins NA, Ruocco RMSA, Zugaib M. Fatores maternos e resultados perinatais no descolamento prematuro da placenta: comparação entre dois períodos. Rev Bras Ginecol Obstet 2006; 28:324-30.

34. Sou SC, Chen WJ, Hsieh WS, Jeng SF. Severe obstetric complications and birth characteristics in preterm or term delivery were accurately recalled by mothers. J Clin Epidemiol 2006; 59:429-35.

Recebido 23/Nov/2011

Versão final reapresentada em 29/Fev/2012 Aprovado em 27/Mar/2012 